\title{
Evidence of fungal co-colonization in cystic fibrosis children chronically colonized with Aspergillus fumigatus \\ M. Noni ${ }^{1}$, A. Katelari ${ }^{1}$, T. Zaoutis ${ }^{2}$, V. Spoulou ${ }^{1}$, A. Kaditís ${ }^{1}$, A. Michos ${ }^{1}$, S. Doudounakis ${ }^{1}$
}

${ }^{1} 1$ st Department of Pediatrics, "Aghia Sophia" Children's Hospital, Athens, Greece

2 Division of Infectious Diseases, The Children's Hospital of Philadelphia, USA

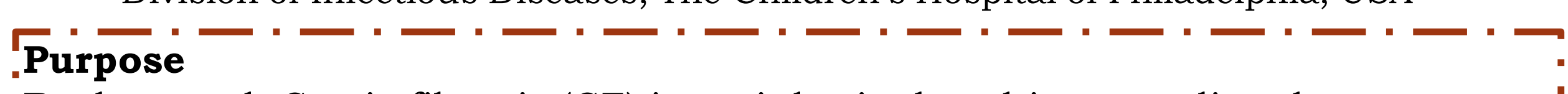

Background: Cystic fibrosis (CF) is an inherited multisystem disorder

characterized mainly by chronic obstructive lung disease. Although bacteria are "considered the primary pathogens, fungi, such as A. fumigatus, have recently gained the attention for their possible negative effect on lung function. Evidence "suggests that mixed colonization indicate a poorer prognosis. The aim of our study was to investigate the microbiota of patients chronically colonized with A. "fumigatus in our CF center.

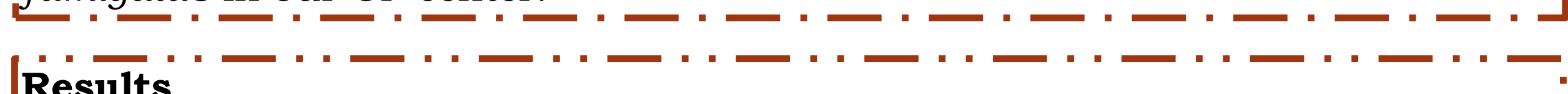

Twenty AC patients matched with 60 controls.

AC patients:10 different types of microorganisms were detected,

Staphylococcus aureus, Pseudomonas aeruginosa, Candida albicans, Aspergillus : "terreus, Stenotrophomonas maltophilia, Candida parapsilosis, Scedosporium apiospermum, Achromobacter xylosoxidans, Aspergillus flavus and Pseudomonas. fluorescens.

* Control patients: 6 different types of microorganisms were detected, S. aureus, P. aeruginosa, C. albicans, Haemophilus influenza, Achromobacter xylosoxidans and Stenotrophomonas maltophilia.

* The presence of $C$. albicans in sputum samples was significantly higher among AC than control patients $(65.0 \%$ vs $23.3 \% ; P=0.001)$, but an independent association was not confirmed from the logistic regression analysis after jadjustment for confounders.

* No significant differences were detected regarding S. aureus and P. aeruginosa : :(70.0\% vs $66.7 \% ; P=0.783$ and $70.0 \%$ vs $50.0 \% ; P=0.119$, respectively).

Figure. Microorganisms detected among CF patients by the time of enrollment in the study

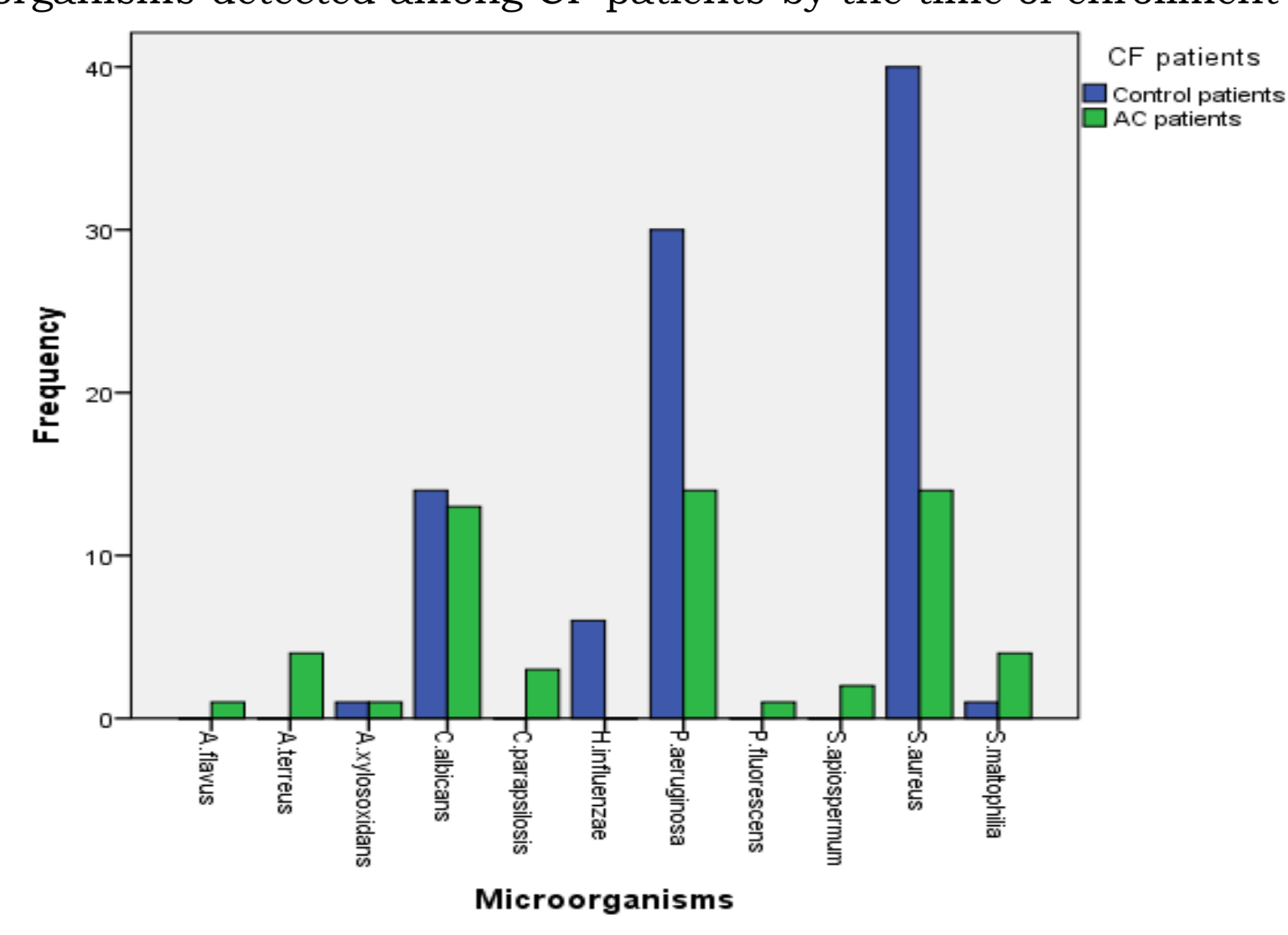

\section{Methods}

A retrospective case-control study of CF patients born from 1989 to 2002 "was performed. Chronic colonization was defined as two or more positive isputum cultures in a given year. Each patient chronically colonized with $A$. fumigatus (AC) was matched with three control $\mathrm{CF}$ patients never colonized:" "with A. fumigatus for age, sex and year of birth ( \pm 3 years). All sputum |cultures from regular visits at the $\mathrm{CF}$ center by the time of enrollment were collected.

Table. Patients' characteristics by the time of enrollment in the study

\begin{tabular}{|c|c|c|c|}
\hline & $\begin{array}{c}\text { Control patients } \\
(\mathrm{n}=60)\end{array}$ & $\begin{array}{c}\text { AC patients } \\
(\mathrm{n}=20)\end{array}$ & $\boldsymbol{P}$ \\
\hline Age (years) & $14[12,15,75]$ & $14[12,15,75]$ & 1,000 \\
\hline $\mathrm{FEV}_{1}(\%$ predicted $)$ & $103,37 \pm 16,87$ & $78,65 \pm 26,74$ & 0,001 \\
\hline BMI $\left(\mathrm{kg} / \mathrm{m}^{2}\right)$ & $20,51 \pm 3,28$ & $19,25 \pm 2,64$ & 0,125 \\
\hline CFRD & $2(3,3)$ & $0(0,0)$ & 1,000 \\
\hline ABPA & $2(3,3)$ & $2(10,0)$ & 0,259 \\
\hline IgE total (IU/mL) & $88,00[24,15201,50]$ & $74,15[34,68280,75]$ & 0,567 \\
\hline $\begin{array}{l}\text { Aspergillus fumigatus specific } \\
\mathrm{IgE}(\mathrm{IU} / \mathrm{mL})\end{array}$ & $0,00[0,001,55]$ & $0,05[0,000,49]$ & 0,824 \\
\hline $\begin{array}{l}\text { Hospitalizations } \\
\text { Patients' microbiota }\end{array}$ & $20(33,3)$ & $17(85,0)$ & $<0,001$ \\
\hline Pseudomonas aeruginosa & $30(50,0)$ & $14(70,0)$ & 0,119 \\
\hline Staphylococcus aureus & $40(66,7)$ & $14(70,0)$ & 0,783 \\
\hline Candida albicans & $14(23,3)$ & $13(65,0)$ & 0,001 \\
\hline \multicolumn{4}{|l|}{ Antibiotic treatment } \\
\hline Inhaled & $38(63,3)$ & $19(95,0)$ & 0,007 \\
\hline & $52(86,7)$ & $18(90,0)$ & 0,696 \\
\hline \multicolumn{4}{|l|}{ Corticosteroid treatment } \\
\hline Inhaled & $9(15,0)$ & $6(30,0)$ & 0,137 \\
\hline Oral & $2(3,3)$ & $2(10,0)$ & 0,259 \\
\hline Azithromycin treatment & $4(6,7)$ & $6(30,0)$ & 0,006 \\
\hline Antifungal treatment & $2(3,3)$ & $3(15,0)$ & 0,097 \\
\hline
\end{tabular}

Categorical data are presented as number (\%). Normally distributed data are presented as mean \pm SD and not normally distributed data as median [interquartile range]. $\mathrm{FEV}_{1}$, Forced expiratory volume in one second BMI, Body mass index; CFRD, Cystic fibrosis-related diabetes; ABPA, Allergic bronchopulmonary aspergillosis.

\section{Conclusión}

" $>$ In our CF center, AC children seem to be co-colonized with C. albicans in high frequency even if this observation, which is in line with our clinical experience, was not confirmed from the statistical analysis.

$>$ The lung microbiota of AC patients merits further study as mixed

colonization may alter the levels or spectrum of virulence factors produced "by bacteria or fungi.

[1] Noni M et.al. Aspergillus fumigatus chronic colonization and lung function decline in cystic fibrosis may have a twoway relationship. Eur J Clin Microbiol Infect Dis. 2015 Nov;34(11):2235-41 